\title{
A Short Review on the Bioactive Constituents from Six Terminalia Species
}

\author{
Mohammad Musarraf Hussain \\ Department of Pharmacy, Faculty of Life and Earth Sciences, Jagannath University \\ Dhaka-1100, Bangladesh
}

(Received: August 18, 2020; Accepted: December 16, 2020; Published (web): January 28, 2021)

\begin{abstract}
Terminalia as a genus has received a great attraction to evaluate and examine the pharmacological potential having their medicinal properties. Different species under Terminalia genus have been used as herbal medicine with various formulations in the treatment of abdominal pain, cancer, cough, conjunctivitis, diarrea, heart problem, leproscopy, urinary tract infection, and sexual related diseases. These properties have been reported to express abundant biological characteristics for example antioxidant, antiparasitic, antibacterial, antifungal, antiviral, and anti-inflammatory. This review has constructed to solicitude the phytochemicals from the genus Terminalia. A total six species belongs to this genus such as Terminalia chebula, T. citrina, T. phanerophlebia, T. belerica, T. catappa, and T. arjuna have been studied and fifty-six phytochemicals with their chemical structures have been reported in this review. Terminalia chebula consists of a higher number of phytochemicals as compared to the other species.
\end{abstract}

Key words: Terminalia, Phytochemicals, Tannins, Glucosides, Pyranosides, Triterpenoids, Phenolic compounds.

\section{Introduction}

Nature is a significant resource for medicinal plants. The medicinal plants along with elucidated chemical compounds having pharmacological activities are using as traditional medicinal agents in the prevention and management of different diseases for many years (Hussain 2020, 2019a, 2019b, 2018). These medicinal plants having varied curative properties like anti-microbial, anticancer, antiinflammatory, anti-plasmodial, and anti-oxidant potentials (Hussain et al., 2016, 2011, 2010; Billah et al., 2013; Ismail et al., 2010). Terminalia is a second prevalent genus under the family of Combretaceae having 200 species and is distributed in tropical countries especially in Southeast Asia (Fahmy et al., 2015). T. chebula is a medium tree, which can go up to $30.0 \mathrm{~m}$ in height, with a trunk having diameter up to $1.0 \mathrm{~m}$. This plant also has traditional use as medicine to treat liver and kidney dysfunction. Dried fruit is also used in Ayurveda as cardiotonic, antitussive, diuretic, homeostatic, laxative, and diuretic (Tawaril et al., 2017).

\section{Reported bio-active constituents}

A total six medicinal plants from Terminalia genus for example Terminalia chebula, T. citrina, $T$. phanerophlebia, T. belerica, T. catappa, and $T$. arjuna, were reviewed and fifty six (1-56; Figures 16) phytoconstituents have been reported along with their chemical structures in this article.

Tannins: Several tannins having hepatitis C inhibitory potentials were isolated from Terminalia chebula, which are chebumeinin A (1) and B (2), chebulic acid (3), casuarinin (4), pentagalloyl glucose (5), 5-O-galloylshikimic acid (6), ethyl

Corresponding author: Mohammad Musarraf Hussain, Email: m.musarraf.hussain@ gmail.com and mmhussain@pharm.jnu.ac.bd

DOI: https://doi.org/10.3329/bpj.v24i1.51638 
gallate (7), gallic acid (8), corilagin (9), chebulagic galloyl- $\beta$-D-glucose (14) (Figure 1) (Ajala et al., acid (10), ellagic acid (11), tetra-o-galloyl- $\beta$-D- 2014; Mahajan et al., 2010; Saleem et al., 2002; Lee glucose (12), chebulinic acid (13), and penta-O- et al., 2007; Han et al., 2006).<smiles>O=C(O)CC(C(=O)O)C1c2c(cc(O)c(O)c2O)C(=O)OC1C(O)C1OC(OC(=O)c2cc(O)c(O)c(O)c2)C(O)C1O</smiles>

1

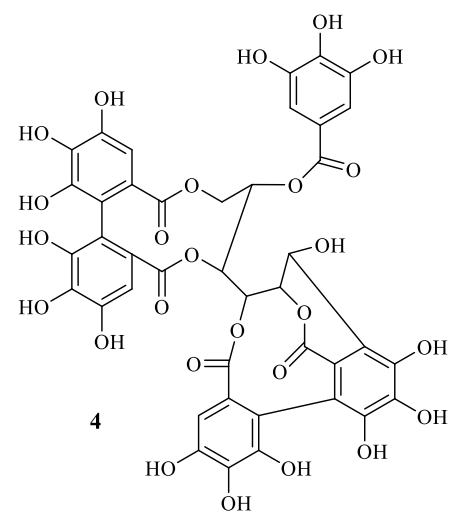

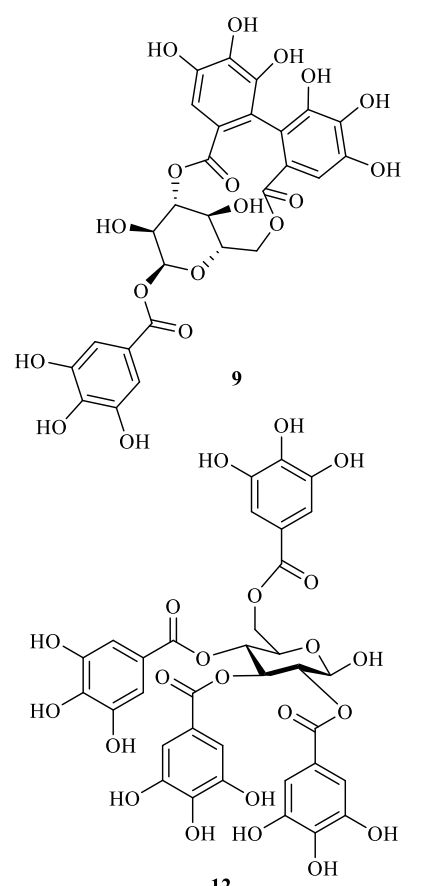

12<smiles>O=C1OC(C(=O)OC(O)C(O)CO)C(C(C(=O)O)C(=O)O)c2c1cc(O)c(O)c2O</smiles><smiles>O=C(O)CC(C(=O)O)C1c2c(cc(O)c(O)c2O)C(=O)OC1C(=O)O</smiles><smiles></smiles><smiles>CCOC(=O)c1cc(O)c(O)c(O)c1</smiles>

6<smiles>O=C(O)c1cc(O)c(O)c(O)c1</smiles>

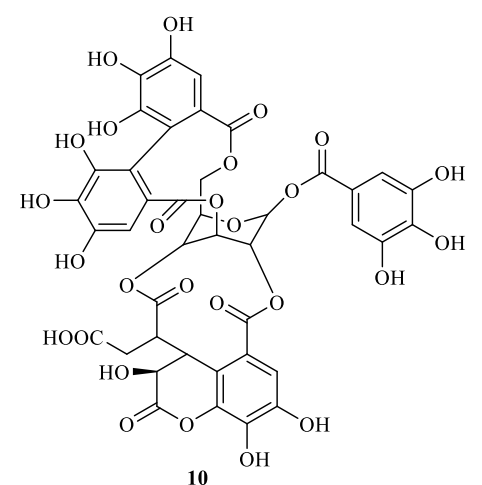<smiles></smiles>

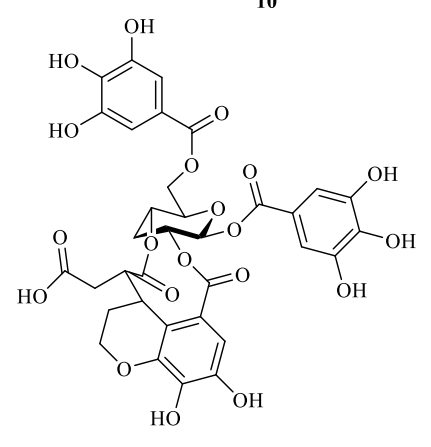

13

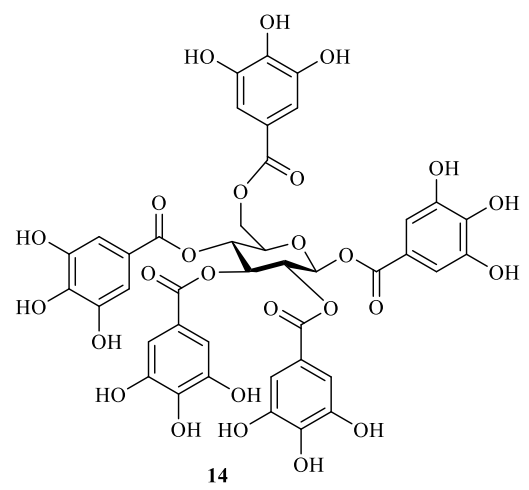

14

Figure 1. Tannins from Terminalia chebula

Glucosides: Six glucosides and one glucosidic ester bearing antiestrogenic and antimicrobial property were elucidated from Terminalia citrine and
Terminalia phanerophlebia; for example, Tterminaloside-L (15), terminaloside-M (16), terminaloside- $\mathrm{N} \quad(\mathbf{1 7}), \quad$ terminaloside-O 
terminaloside-P

(19),

methyl-3,4,5compound 21 was reported to be a lead molecule for trihydroxybenzoate (20), and 1,6-di-o-coumaroyl the discovery of medicinal agents targeting glucopyranoside (21). Among all the isolated tuberculosis (Figure 2) (Muhit et al., 2016; glucosidic compounds from Terminalia genus, Madikizela et al., 2014).<smiles>COc1cc([C@@H]2OC(=O)[C@H]3[C@H](c4cc(OC)c5c(c4)OCO5)OC[C@H]23)cc(OC)c1OC</smiles>

15<smiles>COc1cc([C@@H]2OC[C@@H]3[C@H](c4cc(OC)c(OC)c(OC)c4OC)OC(=O)[C@@H]23)cc2c1OCO2</smiles>

16<smiles>COc1cc([C@@H]2OC[C@H]3[C@H](c4cc(O)c(OC)c(OC)c4OC)OC(=O)[C@H]32)cc2c1OCO2</smiles>

17<smiles>COc1cc([C@@H]2OC[C@@H]3[C@@H]2C(=O)O[C@H]3c2cc(OC)c(OC)c(OC)c2OC)cc2c1OCO2</smiles>

18<smiles>COc1cc([C@@H]2OC[C@@H]3[C@H](c4cc(OC)c(OC)c(OC)c4OC)OC(=O)[C@@H]23)cc2c1OCO2</smiles>

19<smiles>COC(=O)c1cc(O)c(O)c(O)c1</smiles>

20<smiles>O=C(/C=C/c1ccc(O)cc1)OC[C@H]1O[C@H](OC(=O)/C=C/c2ccc(O)cc2)[C@@H](O)[C@H]1O</smiles>

21

Figure 2. Glucosides from Terminalia citrina (15-19) and Terminalia phanerophlebia $(\mathbf{2 0 , 2 1 )}$

Pyranoside: Antiplatelet and antioxidant potency containing one pyranoside, benzoyl- $\beta$-D$\left(4^{\prime} \rightarrow 10^{\prime \prime}\right.$ geranilanoxy)-pyranoside (22), has been reported from Terminalia belerica (Figure 3) (Ansari et al., 2016).

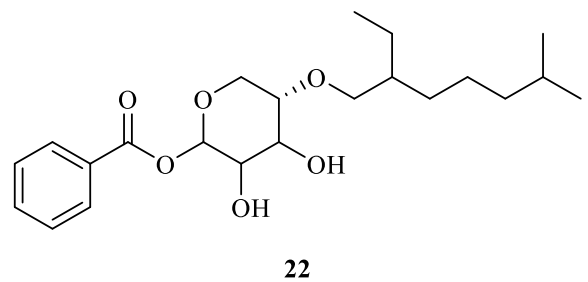

Figure 3. Pyranoside from Terminalia belerica 
Triterpenoids: The reported triterpenoids from the medicinal plant, Terminalia chebula are 28-O- $\beta$ D-glycopyranosyl ester (23), 23-O-4'-epineochebuloylarjungenin (24), arjungenin (25), arjunic acid (26), 23-O-galloylarjunic acid (27), arjunglucoside I (28), arjunglucoside II (29), quercotriter-penoside I (30), terminolic acid (31), 28-
O- $\beta$-D-glucopyranosyl-23-O-galloylterminoliate

(32), arjunolic acid (33), 23-O-galloylarjunolic acid (34), 28-O- $\beta$-D-glucopyranosyl-23-O-galloylarjunolate (35), arjunetin (36), crataegioside (37), 28-O$\beta$-D-glucopyranosyl-pinfaenoate (38), and 28-O- $\beta$-Dglucopyranosyl-23-O-galloylpinfaenoate (39) (Figure 4) (Lee et al., 2017).

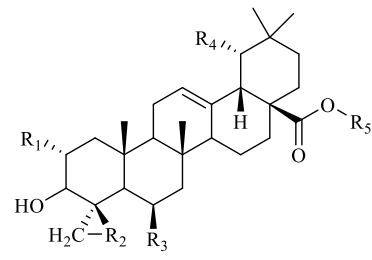

23-37

23: $\mathrm{R}_{1}=\mathrm{OH}, \mathrm{R}_{2}=\mathrm{N}_{1}, \mathrm{R}_{3}=\mathrm{H}, \mathrm{R}_{4}=\mathrm{OH}, \mathrm{R}_{5}=\beta$-D-Glucose 24: $R_{1}=O H, R_{2}=N_{2}, R_{3}=H, R_{4}=O H, R_{5}=H$ 25: $\mathrm{R}_{1}=\mathrm{OH}, \mathrm{R}_{2}=\mathrm{OH}, \mathrm{R}_{3}=\mathrm{H}, \mathrm{R}_{4}=\mathrm{OH}, \mathrm{R}_{5}=\mathrm{H}$ 26: $\mathrm{R}_{1}=\mathrm{OH}, \mathrm{R}_{2}=\mathrm{H}, \mathrm{R}_{3}=\mathrm{H}, \mathrm{R}_{4}=\mathrm{OH}, \mathrm{R}_{5}=\mathrm{H}$ 27: $\mathrm{R}_{1}=\mathrm{OH}, \mathrm{R}_{2}=\mathrm{O}-\mathrm{Gal}, \mathrm{R}_{3}=\mathrm{H}, \mathrm{R}_{4}=\mathrm{OH}, \mathrm{R}_{5}=\mathrm{H}$ 28: $\mathrm{R}_{1}=\mathrm{OH}, \mathrm{R}_{2}=\mathrm{OH}, \mathrm{R}_{3}=\mathrm{H}, \mathrm{R}_{4}=\mathrm{OH}, \mathrm{R}_{5}=\beta$-D-Glucose 29: $R_{1}=O H, R_{2}=O H, R_{3}=H, R_{4}=H, R_{5}=\beta$-D-Glucose 30: $R_{1}=O H, R_{2}=O-G a l, R_{3}=H, R_{4}=O H, R_{5}=\beta$-D-Glucose 31: $\mathrm{R}_{1}=\mathrm{OH}, \mathrm{R}_{2}=\mathrm{OH}, \mathrm{R}_{3}=\mathrm{OH}, \mathrm{R}_{4}=\mathrm{H}, \mathrm{R}_{5}=\mathrm{H}$

32: $\mathrm{R}_{1}=\mathrm{OH}, \mathrm{R}_{2}=\mathrm{O}$-Gal, $\mathrm{R}_{3}=\mathrm{OH}, \mathrm{R}_{4}=\mathrm{H}, \mathrm{R}_{5}=\beta$-D-Glucose 33: $\mathrm{R}_{1}=\mathrm{OH}, \mathrm{R}_{2}=\mathrm{OH}, \mathrm{R}_{3}=\mathrm{H}, \mathrm{R}_{4}=\mathrm{H}, \mathrm{R}_{5}=\mathrm{H}$ 34: $\mathrm{R}_{1}=\mathrm{OH}, \mathrm{R}_{2}=\mathrm{O}-\mathrm{Gal}, \mathrm{R}_{3}=\mathrm{H}, \mathrm{R}_{4}=\mathrm{H}, \mathrm{R}_{5}=\mathrm{H}$ 35: $\mathrm{R}_{1}=\mathrm{OH}, \mathrm{R}_{2}=\mathrm{O}$-Gal, $\mathrm{R}_{3}=\mathrm{H}, \mathrm{R}_{4}=\mathrm{H}, \mathrm{R}_{5}=\beta$-D-Glucose 36: $\mathrm{R}_{1}=\mathrm{OH}, \mathrm{R}_{2}=\mathrm{H}, \mathrm{R}_{3}=\mathrm{H}, \mathrm{R}_{4}=\mathrm{OH}, \mathrm{R}_{5}=\beta$-D-Glucose 37: $\mathrm{R}_{1}=\mathrm{H}, \mathrm{R}_{2}=\mathrm{OH}_{1}, \mathrm{R}_{3}=\mathrm{H}, \mathrm{R}_{4}=\mathrm{OH}, \mathrm{R}_{5}=\beta$-D-Glucose

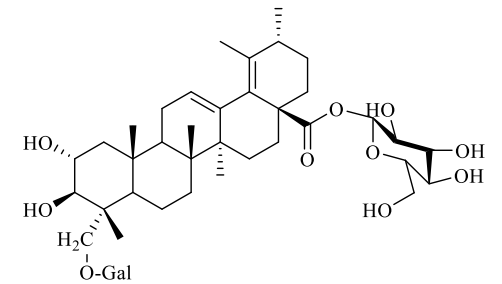

39

38
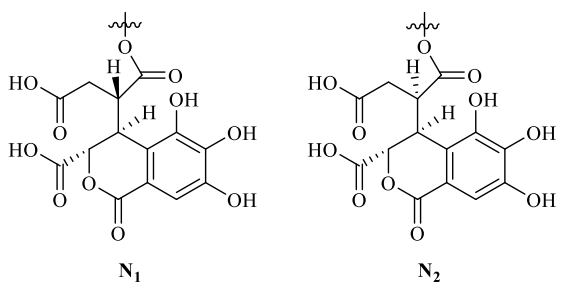<smiles>CC(=O)c1cc(O)c(O)c(O)c1</smiles>

Figure 4. Triterpenoids from Terminalia chebula

Acidic compounds: Five acidic and two other compounds (i.e., carbonyl and o-glucosidic compounds) such as 2-pentadecanone (40), vanillic acid (or, 4-hydroxy-3-methoxybenzoic acid) (41), syringic acid (or, 4-hydroxy-3,5-dimethoxybenzoic acid) (42), ferulic acid (or, (E)-3-(4-hydroxy-3methoxyphenyl)acrylic acid) (43), p-coumaric acid (or, (E)-3-(4-hydroxyphenyl)acrylic acid) (44), 3,4,4'tri-O-methyl-ellagic acid (45), and $\beta$-sitosterol-3-O$\beta$-D-glucoside (46) were elucidated from Terminalia catappa (Figure 5) (Baratelli et al., 2012; Hussain et al., 2016, 2008).

\section{Miscellaneous compounds}

The isolated phytochemicals from Terminalia arjuna are triterpenoids, ursane tri-terpene-glucosyl ester, ursane tri-terpene-glycosyl esters and phenolic compounds. Reported compounds under these classes are arjunic acid (47), arjunetin (48), arjungenin (49),
28 -O- $\beta$-D-glucopyranosyl-2 $\alpha, 3 \beta$-dihydroxyurs12,18-dien-28-oate (50), 28 -O- $\beta$-D-glucopyranosyl$2 \alpha, 3 \beta, 23$-trihydroxyurs-12,18-dien-28-oate quadranoside viii (52), kajiichigoside f1 (53), 28-O$\beta$-D-glucopyranosyl-2 $\alpha, 3 \beta, 23$-trihydroxyurs-12,19dien-28-oate (54), 3-O-methylellagic acid 4'-O- $\alpha-1-$ rhamnopyranoside (55), and (-)-epicatechin (or, (2R,3R)-2-(3,4-dihydroxyphenyl)chromane-3,5,7triol) (56) (Figure 6) (Varghesea et al., 2015; Wang et al., 2010; Toumy et al., 2003).

\section{Concluding remarks and future perspective}

Extracts from the different plants under the genus Terminalia is a good source of phytochemical compounds. A number of reports have revealed ethnopharmacological potential from the different medicinal plants (Das et al., 2020). Here, a short survey has been performed based on the literature on the genus Terminalia which revealed different 


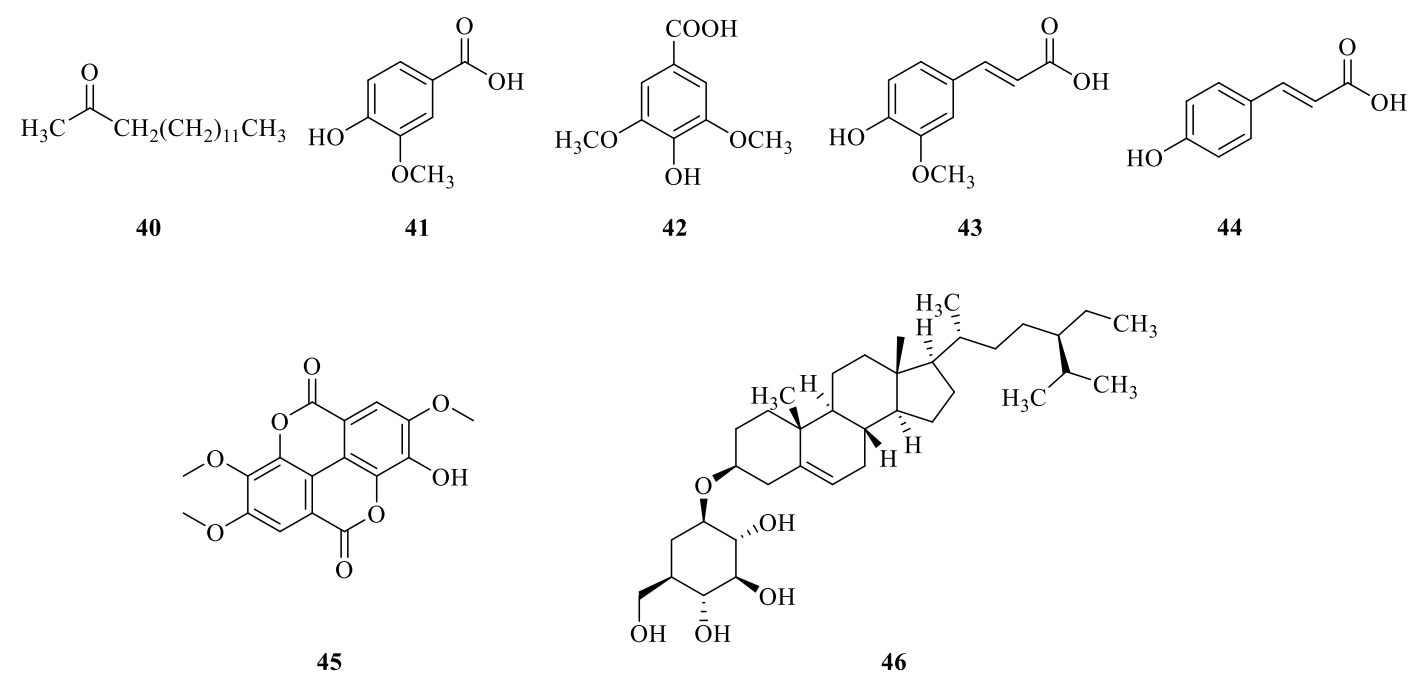

Figure 5. Acidic compounds from Terminalia catappa

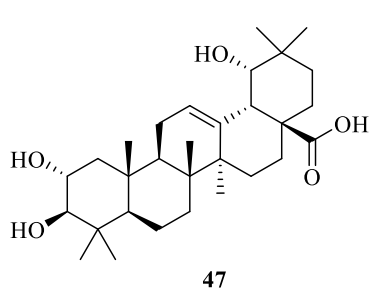

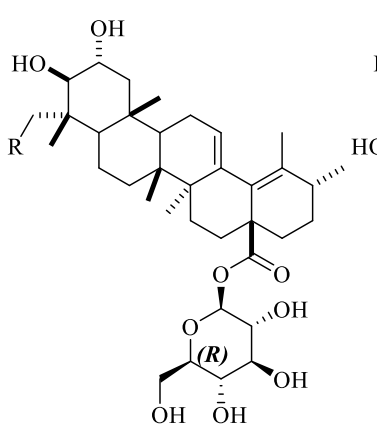

50: $\mathrm{R}=\mathrm{H} ; 49: \mathrm{R}=\mathrm{OH}$

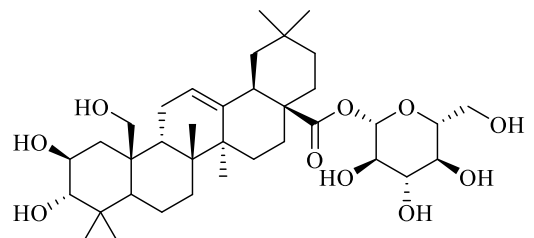

48

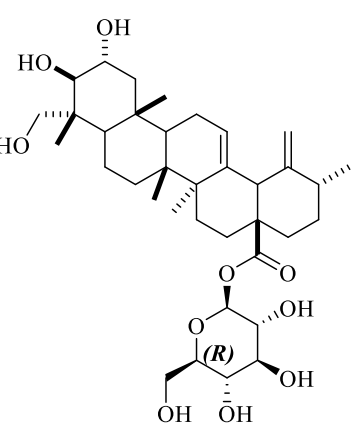

52

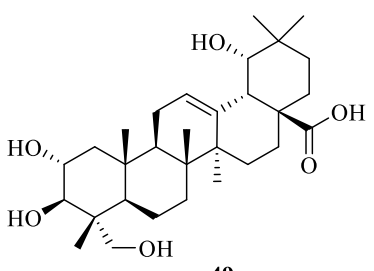

49

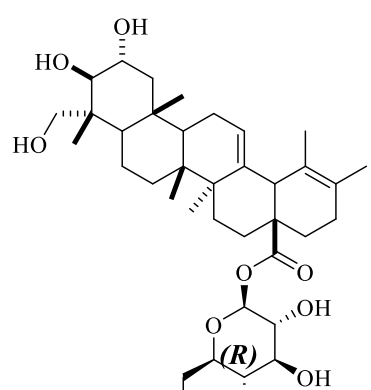

$\mathrm{OH} \quad \overline{\mathrm{O}} \mathrm{H}$<smiles>COc1c(O[C@@H]2O[C@H](C)[C@@H](O)[C@H](O)[C@H]2O)cc2c(=O)oc3c(O)c(O)cc4c(=O)oc1c2c34</smiles>

55

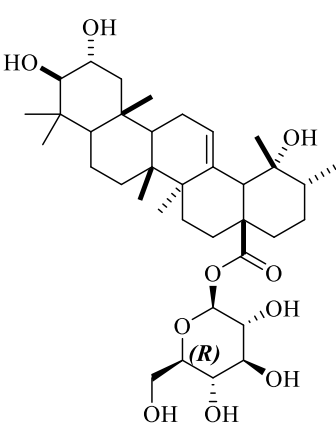

53

54

Figure 6. Compounds from Terminalia arjuna 
phytochemicals such as tannis, glucosides, pyranosides, triterpenoids, and acidic compounds (Fahmy et al., 2015). Six medicinal plants (Terminalia chebula, T. citrina, T. phanerophlebia, T. belerica, $T$. catappa, and T. arjuna) under the genus Terminalia have been studied and fifty six compounds were reported having varied and distinctive chemical structure various bioactivity and pharmacological properties. The current review presents a short perceptive of the chemistry of various Terminalia species, that can be helpful in the progress and finding of the novel medicinal moieties for the diagnosis and treatment of different health related problems and diseases.

\section{References}

Ajala, O.S., Jukov, A., Ma, C.M., 2014. Hepatitis C virus inhibitory hydrolysable tannins from the fruits of Terminalia chebula. Fitoterapia. 99, 117-123.

Ansari, V.A., Arif, M., Hussain, M.S., Siddique, H.H., Dixit, R.K. 2016. New 4 -Substituted benzoyl- $\beta-D$ glycoside from the fruit pulp of Terminalia belerica with antiplatelet and antioxidant potency. Integr. Med. Res. 5, 317-323.

Baratelli, T.D.G., Gomes, A.C.C., Wessjohann, L.A., Kuster, R.M., Siams, N.K. 2012. Phytochemical and allelopathic studies of Terminalia catappa L. (Combretaceae). Biochem. Systematics Ecology 41, 119-125.

Billah, A.H.M.M., Hussain, M.M., Dastagir, M.G., Ismail, M., Quader, A. 2013. $\alpha$-Spinasterol from Amaranthus spinosus stem. Bol. Latinoam. Caribe Plant. Med. Aromat. 12, 15-17.

Das, G., Kim, D-Y., Fan, C.,Gutie’ rrez-Grijalva, E.P., Heredia, J.B., Nissapatorn, V., Mitsuwan, W., Pereira, M.L., Nawaz, M., Siyadatpanah, A., Norouzi, R., Sawicka, B., Shin, H-S., Patra, J.K. 2020. Plants of the genus Terminalia: An insight on its biological potentials, pre-clinical and clinical studies. Front. Pharmacol. 11, 561248.

Fahmy, N.M., Al-Sayed, E., Singab, A.N. 2015. Genus Terminalia: A phytochemical and biological review. Med. Aromat. Plants 4, 218.

Han, Q., Song, J., Qiao, C., Wong, L., Xu, H. 2006. Preparative isolation of hydrolysable tannins chebulagic acid and chebulinic acid from Terminalia chebula by high-speed counter-current chromatography. J. Sep. Sci. 29, 1653-1657.
Hussain, M.M. 2018. A short review on phytoconstituents from the genera Albizzia and Erythrina. Bangladesh Pharm. J. 21, 160-172.

Hussain, M.M. 2019a. A mini review on the chemical compounds of the genus Acacia. Bangladesh Pharm. J. 22, 235-242.

Hussain, M.M. 2019b. A comprehensive review on the phytoconstituents from six species of the genus Amaranthus. Bangladesh Pharm. J. 22, 117-124.

Hussain, M.M. 2020. A further comprehensive review on the phytoconstituents from the genus Erythrina. Bangladesh Pharm. J. 23, 65-77.

Hussain, M.M., Dastagir, M.G., Billah, A.H.M.M., Ismail, M. 2011. Alpinum isoflavone from Erythrina stricta Roxb. Bol. Latinoam. Caribe Plant. Med. Aromat. 10, 88-90.

Hussain, M.M., Mughal, M.M.R., Alam, M.M., Dastagir, M.G., Billah, A.H.M.M. Ismail, M. 2010. Antimicrobial activity of $n$-hexane and Ethyl acetate extracts of Erythrina stricta Roxb. Bangladesh J. Microbiol. 27, 65-66.

Hussain, M.M., Rahman, M.S., Jabbar, A., Rashid, M. A. 2008. Phytochemical and biological investigation of Albizzia lebbek Benth. Bol. Latinoam. Caribe Plant. Med. Aromat. 7, 273-278.

Hussain, M.M., Tahia, F., Rashid, M.A. 2016. Secondary metabolites from some species of Albizzia: A review. Bangladesh Pharm. J. 19, 1-8.

Hussain, M.M., Tuhin, M.T.H., Akter, F., Rashid, M.A. 2016. Constituents of Erythrina-a potential source of secondary metabolites: A review. Bangladesh Pharm. J. 19, 237-253.

Ismail, M., Hussain, M.M., Dastagir, M.G., Billah, M., Quader, A. 2010. Phytochemical and antimicrobial investigation of Luffa cylindrical. Bol. Latinoam. Caribe Plant. Med. Aromat. 9, 327-332.

Lee, D.Y., Yang. H., Kim, H.W., Sung, S.H. 2017. New polyhydroxytriterpenoid derivatives from fruits of Terminalia chebula Retz and their $\alpha$-glucosidase and $\alpha$-amylase inhibitory activity. Bioorg. Med. Chem. Lett. 27, 34-39.

Lee, H-S., Jung, S-H., Yun, B-S., Lee, K-W. Lee. 2007. Isolation of chebulic acid from Terminalia chebula Retz. and its antioxidant effect in isolated rat hepatocytes. Archives Toxicol. 81, 211-218.

Madikizela, B., Aderogba, M.A., Finnie, J.F., Staden, J.V. 2014. Isolation and characterization of antimicrobial compounds from Terminalia phanerophlebia Engl. \& Diels leaf extracts. J. Ethnopharmacol. 156, 228-234. 
Mahajan, A., Pai, N. 2010. Simultaneous isolation and identification of phytoconstituents from Terminalia chebula by preparative chromatography. J. Chem. Pharm. Res. 2, 97-103.

Muhit, M.A., Umehara, K., Noguchi, H. 2016. Five furofuranone lignan glucosides from Terminalia citrina inhibit in vitro E2-enhanced breast cancer cell proliferation. Fitoterapia 113, 74-79.

Saleem, A., Husheem, M., Härkönen, P., Pihlaja, K. 2002. Inhibition of cancer cell growth by crude extract and the phenolics of Terminalia chebula Retz fruit. $J$. Ethnopharmacol. 81, 327-336.

Tewaril, D., Mocan, A., Parvanov, E.D., Sah, A.N., Nabavi, S.M., Huminiecki, L., Ma, Z. F., Lee, Y.Y., Czuk, J.O.H., Atanasov, A.G. 2017. Ethnopharmacological approaches for therapy of jaundice: Part II. Highly used plant species from Acanthaceae, Euphorbiaceae, Asteraceae, Combretaceae, and Fabaceae Families. Frontiers Pharmacol. 8, 519.
Toumy, S.A.A.E., Rauwald, H.W. 2003. Two new allagic acid rhamnosides from Punica granatum heart wood. Planta Med. 69, 682-684.

Varghesea, A., Savaib, J., Panditac, N., Gaudda, R. 2015. In vitro modulatory effects of Terminalia arjuna, arjunic acid, arjunetin, and arjungenin on CYP3A4, CYP2D6 and CYP2C9 enzyme activity in human liver microsomes. Toxicol. Reports 2, 806-816.

Wang, W., Ali, Z., Shen, Y., Li, X-C., Khan, I.A. 2010. Ursane triterpenoids from the bark of Terminalia arjuna. Fitoterapia. 81, 480-484. 